\title{
Forecasting models generation of the electronic means quality
}

\author{
R.O. Mishanov ${ }^{1}$, S.V. Tyulevin ${ }^{2}$, M.N. Piganov ${ }^{1}$, E.S. Erantseva ${ }^{1}$ \\ ${ }^{I}$ Samara National Research University, 34 Moskovskoe Shosse, 443086, Samara, Russia \\ ${ }^{2}$ JSC SRC Progress, 18 Zemetsa street, 443009, Samara, Russia
}

\begin{abstract}
The article describes the results of forecasting models generation of quality and reliability indicators of the electronic means. In the learning process variants of normalizing and centering of controlled parameters are described. Much attention is given to the methods of the Theory of Pattern Recognition and extrapolation methods. This paper gives information about the advanced technique of the models generation and individual forecasting of electronic means for the space equipment. The verification of derived models is investigated in detail. Special emphasis is paid to the analysis of the models efficiency.
\end{abstract}

Keywords: forecasting model; electronic means; verification; learning; informative parameters; analysis

\section{Introduction}

A realization of increasing requirements to the quality and reliability of the radio-electronic means and electronic components (EC) is ensured by the improvement of their design, manufacturing technology, controlling methods and testing. In addition, some hidden defects are not detected by the existing system of technological control and testing methods. The decisive influence on the reliability of hidden defects determines the development of works on the investigation of mechanisms and the causes of failures. However, a special interest is caused by using methods and means of flaw detection and physicochemical analysis.

Despite the effectiveness of work in this direction, the complexity and high cost of their implementation caused the necessity to search for and develop methods and means to identify hidden defects of the EC, which correspond to the pace of modern batch production. In addition, about $30 \%$ of defects and failures of EC cannot be controlled by these methods and means [1].

Thus, methods of testing and forecasting reliability and other quality indicators based on the informative parameters are being developed [2-8], which are reposed on the assumption of the existence of a stochastic connection between reliability and initial values of the informative parameters set of the product. The choice of the informative parameters set has a decisive influence on the validity of testing and forecasting. Ensuring the presence of informative parameters in the initial set is assigned to the researcher and in most cases is a very difficult task.

Ensuring the quality and reliability of space electronics requires a wide implementation of new methods of diagnostic nondestructive testing (NDT) [9-15]. For their development, it is necessary to establish the dependencies of the main reliability indicators on the physical properties and parameters of the devices, on the physicochemical processes occurring in them, and on the physical nature of the failures mechanisms [16].

One of the promising directions in the development of effective and economically acceptable methods for assessing the quality and reliability is to forecast their future state.

Forecasting failures of the devices can be carried out at various stages of their life cycle (control, testing, application, operation). The individual forecasting (IF) provides the greatest accuracy. Its meaning is to estimate the potential reliability of each instance using the forecasting model and information about the value of the informative parameter or results of monitoring the instances [17]. A structural IF model is required to generate an operator (mathematical model), an algorithm, an individual forecasting technique, and a hardware quality management. Such a model is generated in the form of an enlarged technological scheme with a description of the functions performed by the component parts [18].

A new structural forecasting model was proposed to increase the accuracy of the IF. It includes the following interrelated steps:

- analysis of the IF methods;

- physical and technical analysis of the failures;

- preliminary selection of the informative parameters and selection of the forecasting parameters;

- development of the investigation test technique;

- learning experiment;

- final selection of the informative parameters;

- selection of the IF method;

- algorithm development;

- program development;

- evaluation of the software product quality;

- development of the forecast model (the IF operator);

- evaluation of the IF operator models quality;

- development of working technique;

- verification of the model; 
- attestation of the technique;

- operational forecasting;

- optimization of the model;

- refinement of the IF model;

- clarifying learning experiment;

- development or selection of new informative parameters;

- definition of levels;

- development of the recommendations;

- technological process (TP);

- parameter checkout of the radio-electronic means;

- change of the design and technology option;

- refinement of the technique;

- verification of the updated technique;

- heuristic forecasting or a rejection.

\section{Development of the IF operators based on the regression models}

The IF task including the value estimation of the forecasting parameter with a large number of the informative parameters was solved using the regression models. A problem statement was reduced to the determination of the operator $H_{x}$.

When the linear model of the connection between $\tilde{y}$ and $x_{i}$ is adopted the estimation of the forecasting parameter value of the $\mathrm{j}^{\text {th }}$ element is defined by [19]:

$$
y^{*(j)}\left(t_{f}\right)=H_{x}\left[\left\{x_{i}^{(j)}\right\}\right]=B_{0}+B_{1} x_{1}^{(j)}+B_{2} x_{2}^{(j)}+\cdots+B_{i} x_{i}^{(j)}+\cdots+B_{k} x_{k}^{(j)},
$$

where $x_{i}^{(j)}$ - the value of the $\mathrm{i}^{\text {th }}$ attribute of the $\mathrm{j}^{\text {th }}$ element; $B_{i}$ - constant coefficients.

To find the coefficients $B_{i}$ in a linear regression model, it is more convenient to turn the initial data to the centered and normalized values $\tilde{x}_{i c}$, which were determined by:

$$
\tilde{x}_{i c}=\frac{\tilde{x}_{i}-M^{*}\left[\tilde{x}_{i}\right]}{D^{* 1 / 2}\left[\tilde{x}_{i}\right]} .
$$

$M^{*}\left[x_{i}\right]$ and $D^{*}\left[x_{i}\right]$ are the estimates of the expected value and standard deviation of the random variable $\tilde{x}_{i}$ calculated from the learning experiment data:

$$
\begin{aligned}
& M^{*}\left[\tilde{x}_{i}\right]=\frac{1}{n} \sum_{j=1}^{n} x_{i}^{(j)} \\
& D^{* 1 / 2}\left[\tilde{x}_{i}\right]=\sqrt{\frac{1}{n-1} \sum_{j=1}^{n}\left(x_{i}^{(j)}-M^{*}\left[\tilde{x}_{i}\right]\right)^{2} .}
\end{aligned}
$$

The idea of representing the connection between the forecasting parameter and informative parameters in the form of a regression model is as follows [20].

The coefficients $b_{i}$ always can be found for any centered and normalized values $\tilde{y}_{i c}$ and $\tilde{x}_{i c}$ while the equation (2) has meaning regardless of the distribution law of random variables.

$$
\tilde{y}_{c}=b_{1} \tilde{x}_{1 c}+b_{2} \tilde{x}_{2 c}+\cdots+b_{k} \tilde{x}_{k c}+\Delta \tilde{y},
$$

In this equation $b_{i}$ are the constant coefficients of the regression model with centered and normalized values of the random variables; $\Delta \tilde{y}-$ a forecasting error.

If the values of the coefficients $b_{i}$ are found, the estimation of the forecasting parameter value can be determined from the expression (2). The coefficients $b_{i}$ must be such that the error variance $D[\Delta \tilde{y}]$ is minimal, and the expected value of the error $M[\Delta \tilde{y}]$ equals zero, i. e.

$$
D[\Delta \tilde{y}] \rightarrow \min , \quad M[\Delta \tilde{y}]=0 .
$$

If the error variance does not exceed the allowable value, the forecasting operator can be recommended to estimate the value of the forecasting parameter of new instances. In this case, having measured the values of its characteristics for the $\mathrm{m}^{\text {th }}$ instance and substituting them into expression (1), we obtain the estimate:

$$
y^{*(m)}\left(t_{f}\right)=B_{0}+B_{1} x_{1}^{(m)}+B_{2} x_{2}^{(m)}+\cdots+B_{k} x_{k}^{(m)} .
$$

The estimation of the forecasting error will be more accurate than the larger sample size is used in learning experiment. In this case the estimates of the expectation value, the standard deviation and the correlation coefficient will be found more accurately. For CMOS chips and stabilitrons the forecasting operators were obtained (Table 1).

Table 1 . The forecasting models of study samples.

\begin{tabular}{cc} 
Number of sample & Forecasting model (IF operator) \\
\hline Sample №44 & $\frac{\Delta I_{l c}}{I_{l c}}=-29,53+29,11 t_{p}^{+}-51,07 U_{s}$ \\
\hline
\end{tabular}

Sample №45 $\Delta U_{s}=-46,94+42,04 K_{T}+0,096 R_{d}$ 


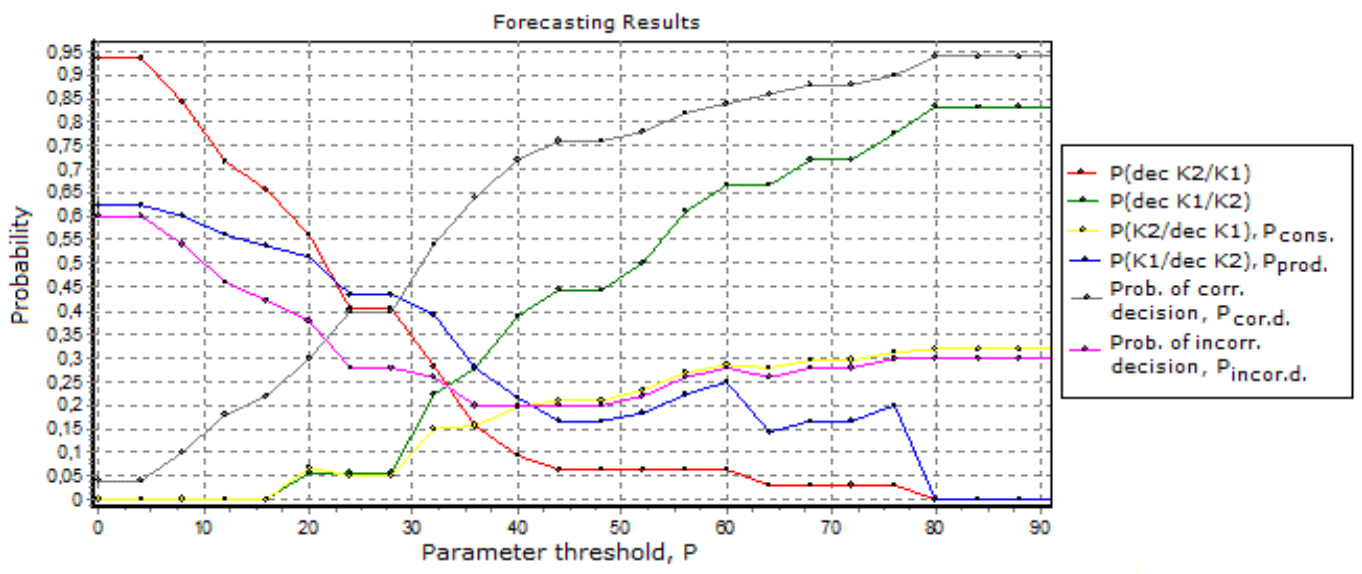

Fig. 1. The dependence of the probabilistic characteristics on the threshold $P$ of the regression function of the CMOS chips.

$\Delta I_{l c} / I_{l c}-$ a leakage current drift, $t_{p}^{+}-$a rise time of the signal, $U_{s}-$ a supply voltage, $\Delta U_{s}-$ a stabilized voltage drift, $K_{T}-$ a temperature coefficient of stabilization, $R_{d}-$ a differential resistance.

Figure 1 shows the influence of the threshold $P$ on the forecasting efficiency of the CMOS chips.

The analysis of this model have shown that the forecasting operator for the CMOS chips provides the optimal value of the forecasting indicators at the threshold $P=35$. In this case the risk of the incorrect decision $P_{i n c . d}$ equals 0,22 ; Consumer's risk ( $\beta$-Risk) $P_{\text {cons. }}$ equals 0,18 ; Producer's risk ( $\alpha$-Risk) $P_{\text {prod. }}$ equals 0,13 . The minimum value of the $P_{\text {cons. }}$ equals 0 when $P=$ $0 \ldots 16, P_{\text {inc.d }}=0,6 \ldots 0,42 ; P_{\text {prod. }}=0,63 \ldots 0,54$. The minimum value of the $P_{\text {prod. }}$ equals 0 when $P=80 \ldots 90, P_{\text {inc.d }}=0,3 ; P_{\text {cons. }}=$ $0,32 \ldots 0,33$.

Figure 2 shows the influence of the threshold $P$ on the forecasting efficiency of the stabilitrons.

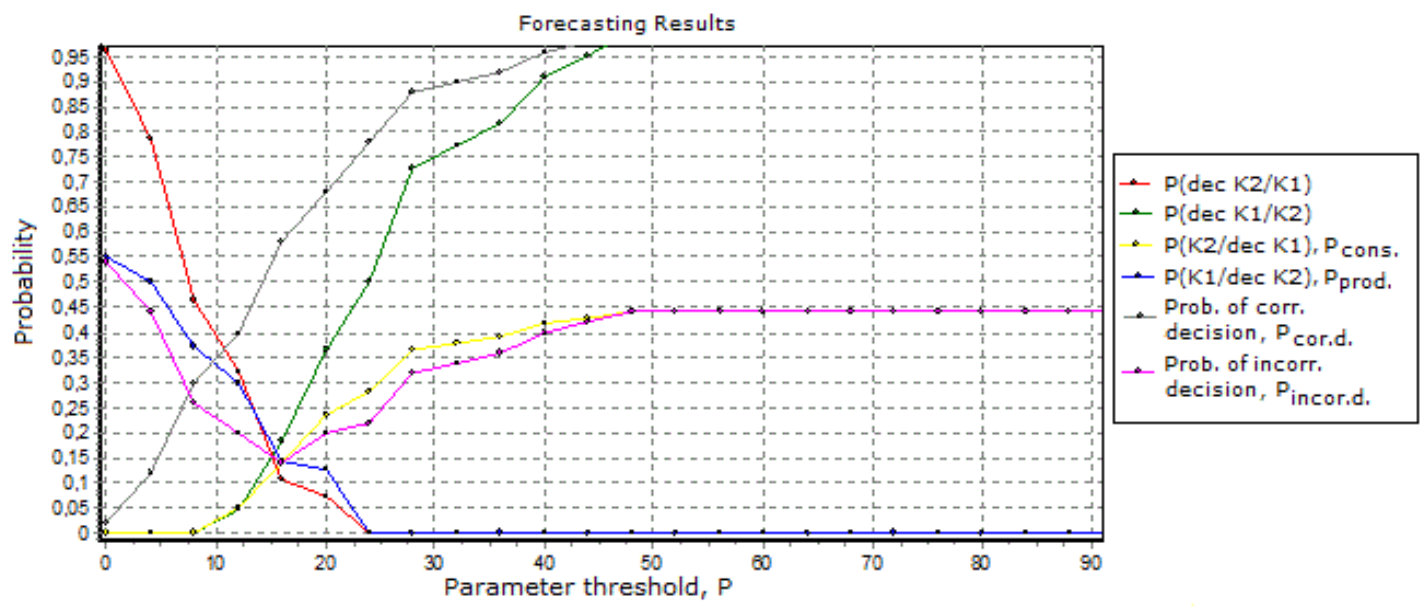

Fig. 2. The dependence of the probabilistic characteristics on the threshold $\mathrm{P}$ of the regression function of the stabilitrons.

The analysis of this model have shown that the forecasting operator for the stabilitrons provides the optimal value of the forecasting indicators at the threshold $P=16$. In this case the risk of the incorrect decision $P_{\text {inc.d }}$ equals 0,15 ; Consumer's risk ( $\beta$-Risk) $P_{\text {cons. }}$ equals 0,14 ; Producer's risk ( $\alpha$-Risk) $P_{\text {prod. }}$ equals 0,14 . The minimum value of the $P_{\text {cons. }}$ equals 0 when $P=$ $0 \ldots 8, P_{\text {inc.d }}=0,54 \ldots 0,26 ; P_{\text {prod. }}=0,55 \ldots 0,37$. The minimum value of the $P_{\text {prod. }}$ equals 0 when $P=24 \ldots 90, P_{\text {inc.d }}=0,22 \ldots 0,44$; $P_{\text {cons. }}=0,29 \ldots 0,44$.

\section{The models verification}

The method of discriminant functions was used for the models verification.

In general terms the problem formulation of such forecasting reduces to find the operator $H_{x c l}$. It is desirable to have the simplest model, when the hyperplane is a surface that divides the space into two regions.

The equation of the (k-1)-dimensional hyperplane in the k-dimensional feature space has the form:

$$
g\left(x_{1}, x_{2}, \ldots, x_{k}\right)=B_{1} x_{1}+B_{2} x_{2}+\cdots+B_{k} x_{k}=P_{d},
$$

where $\boldsymbol{P}_{\boldsymbol{d}}, \boldsymbol{B}_{1}, \boldsymbol{B}_{2}, \ldots, \boldsymbol{B}_{\boldsymbol{k}}$ - constant coefficients that define the position of the hyperplane in the k-dimensional space.

Then the discriminant function takes the form:

$$
g\left(x_{1}, x_{2}, \ldots, x_{k}\right)=B_{1} \tilde{x}_{1}+B_{2} \tilde{x}_{2}+\cdots+B_{k} \tilde{x}_{k} .
$$

In this function the dimension of the coefficients $B_{i}$ is inverse to the dimension of the corresponding characteristics $\tilde{x}_{i}$. 
It was required to find those values of the coefficients $P_{d}$ and $B_{i}$, which in the best way (in the sense of a misclassifications minimum) would specify the position of this hyperplane in the feature space. Since the sample size is limited the estimates $\beta_{i}$ were determined.

The following approach was used to find the estimates of the coefficients $\beta_{i}$. According to the learning experiment, the actual class is known, to which each of $n$ copies belongs $-K_{s}^{(j)}$. It is possible to find the estimates of conditional expected value and conditional variance of each $\mathrm{i}^{\text {th }}$ attribute $x_{i}$ :

$$
\begin{aligned}
M^{*}\left[\tilde{x}_{i} / K_{1}\right] & =\frac{1}{n_{1}} \sum_{\substack{j=1 \\
j \in K_{1}}}^{n_{1}} x_{i}^{(j)}, \\
D^{*}\left[\tilde{x}_{i} / K_{1}\right] & =\frac{1}{n_{1}-1} \sum_{\substack{j=1 \\
j \in K_{1}}}^{n_{1}}\left\{x_{i}^{(j)}-D\left[\tilde{x}_{i} / K_{1}\right]\right\}^{2}, \\
M^{*}\left[\tilde{x}_{i} / K_{2}\right] & =\frac{1}{n_{2}} \sum_{\substack{j=1 \\
j \in K_{2}}}^{n_{2}} x_{i}^{(j)}, \\
D^{*}\left[\tilde{x}_{i} / K_{2}\right] & =\frac{1}{n_{2}-1} \sum_{\substack{j=1 \\
j \in K_{2}}}^{n_{2}}\left\{x_{i}^{(j)}-D\left[\tilde{x}_{i} / K_{2}\right]\right\}^{2} .
\end{aligned}
$$

$n_{1}$ and $n_{2}$ - number of the instances, which belong to the class $K_{1}$ and $K_{2}$, respectively, so that $n_{1}+n_{2}=n$.

Using theorems on the numerical characteristics of random variables, the estimates of the conditional expected values of random variable were determined as:

$$
G=g\left(\tilde{x}_{1}, \tilde{x}_{2}, \ldots, \tilde{x}_{k}\right) \text {. }
$$

If the instance belongs to the class $K_{1}$ :

$$
M^{*}\left[G / K_{1}\right]=\sum_{i=1}^{k} \beta_{i} M^{*}\left[\tilde{x}_{i} / K_{1}\right]
$$

and to the class $K_{2}$ :

$$
M^{*}\left[G / K_{2}\right]=\sum_{i=1}^{k} \beta_{i} M^{*}\left[\tilde{x}_{i} / K_{2}\right]
$$

If the attributes are not correlated the corresponding estimates of conditional variances are equal:

$$
\begin{aligned}
& D^{*}\left[G / K_{1}\right]=\sum_{i=1}^{k} \beta_{i}{ }^{2} D^{*}\left[\tilde{x}_{i} / K_{1}\right] ; \\
& D^{*}\left[G / K_{2}\right]=\sum_{i=1}^{k} \beta_{i}{ }^{2} D^{*}\left[\tilde{x}_{i} / K_{2}\right] ;
\end{aligned}
$$

If the classes are well separated, then $M^{*}\left[G / K_{1}\right]$ and $M^{*}\left[G / K_{2}\right]$ will differ significantly, i.e. $D^{*}\left[G / K_{1}\right]$ and $D^{*}\left[G / K_{2}\right]$ are small. Therefore, as an optimization criterion for finding estimates of the coefficients $\beta_{i}$, we used an expression of the form:

$$
\frac{M^{*}\left[G / K_{1}\right]-M^{*}\left[G / K_{2}\right]}{\sqrt{D^{*}\left[G / K_{1}\right]+D^{*}\left[G / K_{2}\right]}} \rightarrow \text { extr. }
$$

After substituting in the expression (7) the estimates of the conditional expected values and conditional variances of the random variable $G$, determined by the expressions (3) - (6), we obtain the function:

$$
V\left(\beta_{1}, \ldots, \beta_{k}\right)=\left|\frac{\sum_{i=1}^{k} \beta_{i} M^{*}\left[\tilde{x}_{i} / K_{1}\right]-\sum_{i=1}^{k} \beta_{i} M^{*}\left[\tilde{x}_{i} / K_{2}\right]}{\sqrt{\sum_{i=1}^{k} \beta_{i}^{2} D^{*}\left[\tilde{x}_{i} / K_{1}\right]-\sum_{i=1}^{k} \beta_{i}{ }^{2} D^{*}\left[\tilde{x}_{i} / K_{2}\right]}}\right| .
$$

Taking partial derivatives $\partial V / \partial \beta_{i}$ and equating them to zero, we obtain a system of $k$ algebraic equations with $k$ unknown coefficients $\beta_{1}, \beta_{2}, \ldots, \beta_{k}$ for finding optimal estimates $\beta_{i}$ opt . The obtained coefficients $\beta_{i}$ opt will determine the best slope of the hyperplane in the feature space.

Then we find the threshold value $P_{d}$ for the discriminant function $g\left(x_{1}, x_{2}, \ldots, x_{k}\right)$, which specifies the best position of the separating hyperplane. Obviously, the following condition must be satisfied:

$$
\begin{gathered}
M^{*}\left[G / K_{1}\right]>P_{d}>M^{*}\left[G / K_{2}\right] \\
\text { or } \\
M^{*}\left[G / K_{1}\right]<P_{d}<M^{*}\left[G / K_{2}\right] .
\end{gathered}
$$

When the threshold is changed, the risk of the incorrect decisions will change. The value of the threshold was found by several recalculations of the probability of incorrect decisions from the data of the learning experiment for various $P_{d}$ and by choosing one of them at which the risk of incorrect decisions turned out to be the least.

If the obtained risk does not exceed the permissible value, the previously found operator can be used forecast the class of new instances (which not participating in the learning experiment). For this, the values of the attributes $x_{i}^{(m)}$ of the new $\mathrm{m}^{\text {th }}$ instance are measured and the discriminant function has the form:

$$
G^{(m)}=g\left(x_{1}{ }^{(m)}, x_{2}{ }^{(m)}, \ldots, x_{k}{ }^{(m)}\right)=\sum_{i=1}^{k} \beta_{i} x_{i}^{(m)} .
$$


Mathematical Modeling / R.O. Mishanov, S.V. Tyulevin, M.N. Piganov, E.S. Erantseva

If $M^{*}\left[G / K_{1}\right]>M^{*}\left[G / K_{2}\right]$ and $G^{(m)} \geq P_{d}$, then a decision is to relegate the $\mathrm{m}^{\text {th }}$ instance to the class $K_{1}, G^{(m)}<P_{d}$, then a decision is to relegate it to the class $K_{2}$.

The method of discriminant functions made it possible to obtain the forecasting operators (Table 2):

Table 2. The forecasting models of study samples.

\begin{tabular}{cc} 
Number of sample & Forecasting model (IF operator) \\
\hline Sample №44 & $P_{d}=\frac{\Delta I_{l c}}{I_{l c}}+0,76 t_{p}^{+}+0,5 U_{s}$ \\
\hline Sample №45 & $P_{d}=\Delta U_{s}+0,75 K_{T}+0,28 R_{d}$
\end{tabular}

Figure 3 and 4 show the dependencies of the probabilistic characteristics on the discriminant function threshold $P_{d}$ for the CMOS chips and stabilitrons.

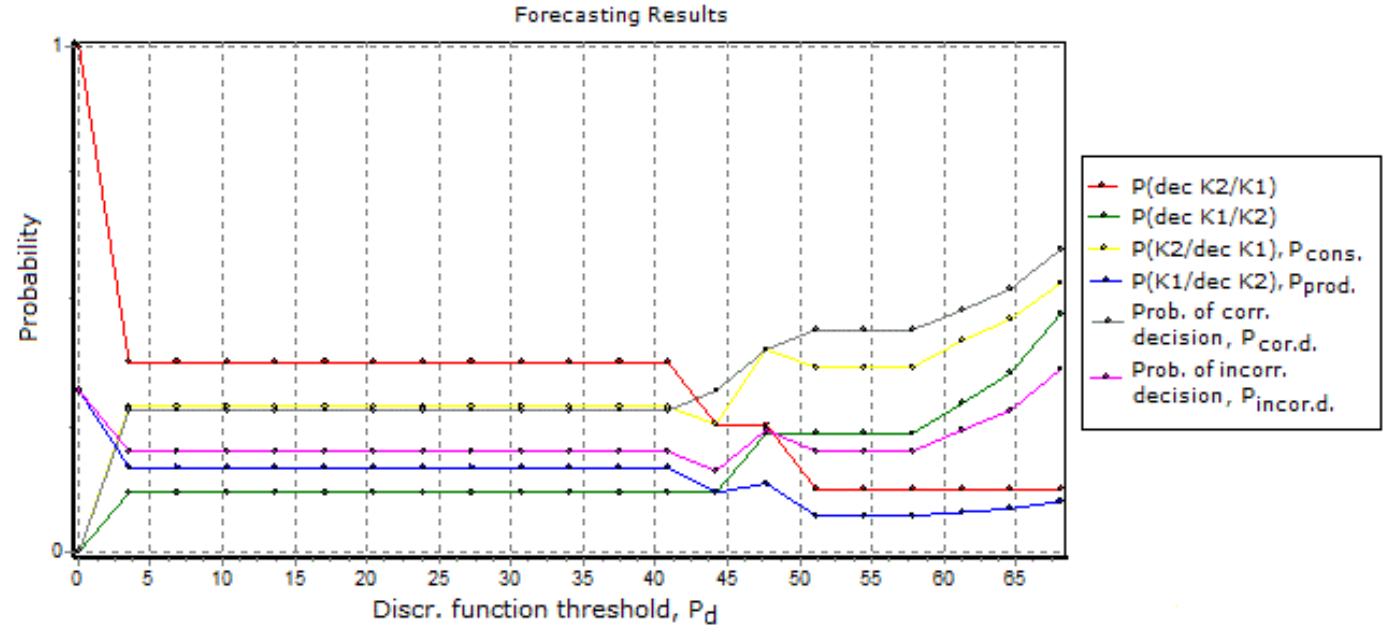

Fig. 3. The influence of the threshold $P_{d}$ on the performance characteristics of the IF operator for the CMOS chips.

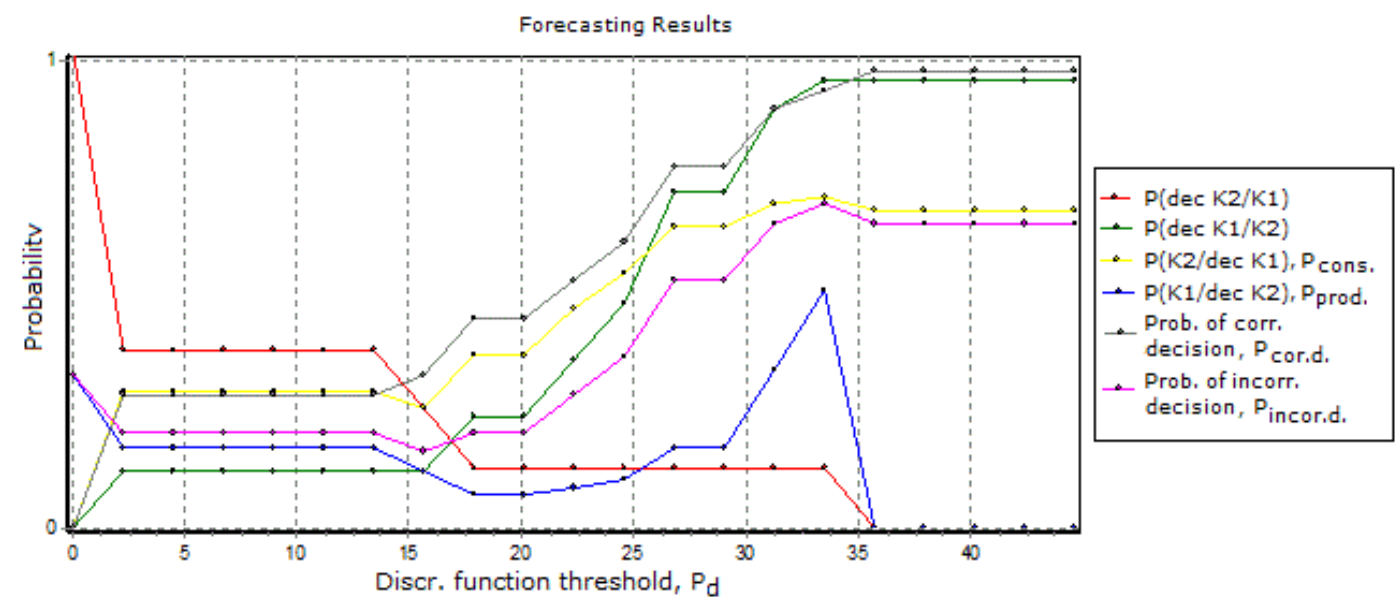

Fig. 4. The influence of the threshold $P_{d}$ on the performance characteristics of the IF operator for the stabilitrons.

The optimal values of the forecasting indicators for the CMOS chips are at the threshold $P_{d}=44$. In this case the risk of the incorrect decision $P_{\text {inc. }}=0,17$; Consumer's risk $\left(\beta\right.$-Risk) $P_{\text {cons. }}=0,27$; Producer's risk $\left(\alpha\right.$-Risk) $P_{\text {prod. }}=0,13$. The minimum value of the $P_{\text {cons. }}$ equals 0,27 when $P_{d}=44$. The minimum value of the $P_{\text {prod. }}$ equals 0 when $P_{d}=57 ; P_{\text {inc.d }}=0,21 ; P_{\text {cons. }}=$ 0,37 .

The optimal values of the forecasting indicators for the stabilitrons are at the threshold $P_{d}=16$. In this case the risk of the incorrect decision $P_{\text {inc. }}=0,18$; Consumer's risk $\left(\beta\right.$-Risk) $P_{\text {cons. }}=0,25$; Producer's risk $\left(\alpha\right.$-Risk) $P_{\text {prod. }}=0,13$. The minimum value of the $P_{\text {cons. }}$ equals 0,25 when $P_{d}=16$. The minimum value of the $P_{\text {prod. }}$ equals 0 when $P_{d} \geq 36 ; P_{\text {inc.d }}=0,52 ; P_{\text {cons. }}=$ 0,57 .

\section{Conclusion}

The method of regression models was chosen for the forecasting models generation of the spacecraft electronic means. The CMOS chips and the stabilitrons were used as the electronic means. The forecasting models allow to provide the IF with the probability of correct decisions $P_{\text {cor.d }}=0,78$ for the chips and $P_{\text {cor.d }}=0,85$ for the stabilitrons. The method of discriminant functions was used to verify obtained models. They gave close to the initial models probabilities of the incorrect decisions: for the chips $P_{\text {inc.d }}=0,22$ and 0,17 ; for the stabilitrons $P_{\text {inc.d }}=0,15$ and 0,18 . 


\section{References}

[1] Berezhnoy VP, Yusov YP, Khodnevich SP. Electrophysical diagnosis of the elements of radio electronic means. Moscow: CRI Electronica Publisher, $1990 ; 304 \mathrm{p}$.

[2] Zhadnov V. Reliability forecasting of electronic means with mechanical elements. Ekaterinburg: Publishing house of LCC Fort-Dialog-Iset, 2014; 172 p.

[3] Tyulevin SV, Piganov MN, Erantseva ES. To the problem of forecasting the quality indices of spacecraft elements. Reliability and quality of complex systems 2014; 1(5): 9-17.

[4] Berenshtein GV, Dyachenko AM. Quality forecasting of the microcircuits based on the analysis of the internal stress. Physical basis of reliability and degradation of semiconductor devices. Chisinau 1991; 4: 36 .

[5] Luchino AI, Savina AS. Possibility investigation of the individual forecasting of the transistor long-eternity by the method of pattern recognition. Electronic Technology 1976; 10: 3-9.

[6] Mishanov RO, MN. Piganov. Individual forecasting of quality characteristics by an extrapolation method for the stabilitrons and the integrated circuits The experience of designing and application of CAD systems in Microelectronics (CADSM 2015): Proceeding XIII international conference. Ukraine, Lviv, 2015; 242-244.

[7] Piganov MN, Tyulevin SV, Erantseva ES. Individual prognosis of quality indicators of space equipment elements. The experience of designing and application of CAD systems in microelectronics (CADSM 2015): Proceeding XIII international conference. Ukraine, Lviv, 2015 ; 367-371.

[8] Mishanov RO, Piganov MN. Generation of the forecasting quality model of the semiconductor devices by extrapolation. Proceedings of the Samara Scientific Center of the Russian Academy of Sciences 2014; 6(4/3): 594-599.

[9] Mishanov RO, Piganov MN. Technology of diagnostic for non-destructive control of the bipolar integrated circuits. Sense. Enable. Spitse: proceedings of the $2^{\text {nd }}$ international scientific symposium. Russia, St. Peterburg, 2015; 38-41.

[10] Sergeev VA, Yudin VV. Quality control of the digital integrated circuits by the parameters of the thermal bond matrix. Proceedings of the Institution of Higher Education. Electronics 2009; 6: 72-78.

[11]Piganov MN, Tyulevin SV, Erantseva ES, Mishanov RO. Apparatus diagnostic for non-destructive control chip CMOS-Type. European science and technology: materials of the VIII international research and practice conference. Germany: Munich, 2014; 398-401.

[12] Watchik R, Bucelot T, Li G. J. Appl. Phys. 1998; 9: 4734-4740.

[13] Jonson JB. The Schottky effect in box frequency circuit. Phys. rev, 1925; 26: 71-85.

[14]Chang MH, Das D, Varde PV, Pecht M. Light emitting diodes reliability review. Microelectronics Reliability 2012; 5: 762-782.

[15]Kuba J. Applicaion of low temperature infailure diagnostics of semiconductor devices. Power Semic. Hybrid Device - th Jnt. Spring Semin. Electrotechnol. Prenet, 1985; 31-34.

[16] Pryanikov VS. Forecasting failures of semiconductor devices. Moscow: Energy, 1978; 122 p.

[17] Piganov MN, Tyulevin SV. The reliability forecasting of the radio-electronic means. St. Petersburg State Polytechnical University Journal. Computer Science. Telecommunication and Control System 2009; 1: 175-182.

[18] Tyulevin SV, Piganov MN. Structural model of the individual forecasting of space equipment parameters. Vestnik of Samara State Aerospace University 2008; 1: 92-96.

[19]Piganov MN. Technological fundamentals of quality assurance of microassemblies. Samara: SSAU Publishing house, $1999 ; 231$.

[20] Tyulevin SV. Method of the individual forecasting of the space radio-electronic means reliability. Actual problems of radio-electronics and telecommunications: materials of the Russian Scientific and Technical Conference. Samara: SSAU Publishing house, 2007; $162-163$. 